\section{A preguiça como explicação da inatividade física: comentários e reflexões sobre discrepâncias entre as evidências científicas e o discurso jornalístico}

\author{
Laziness as the single explanation for physical \\ inactivity: commentary and reflections on \\ discrepancies between scientific evidence \\ and media discourse
}

La pereza como explicación de la inactividad física: comentarios y reflexiones sobre las discrepancias entre las evidencias científicas y el discurso periodístico

\section{Resumo}

Este ensaio busca apresentar elementos para a reflexão sobre discrepâncias entre as evidências científicas produzidas a respeito dos fatores associados e determinantes da inatividade física e as informações que são passadas pela mídia a respeito desse assunto, mais especificamente em um caso em que a "preguiça" foi utilizada como fator "explicativo" para os elevados níveis de inatividade física na população brasileira. Utilizamos, como exemplos, um artigo científico publicado na Nature e duas reportagens veiculadas em canais de comunicação brasileiros de grande alcance (revista Veja e jornal O Globo), que o repercutiram. Foram observadas importantes incoerências entre o conteúdo do artigo da Nature e os títulos das reportagens analisadas, de forma que essas simplificaram um problema real e complexo (os altos níveis de inatividade física no Brasil), dando a entender que a "preguiça" seria o motivo para o Brasil estar mal posicionado em um ranking internacional de prática de atividade física. Destacamos a necessidade de os meios de comunicação em massa buscarem uma melhor contextualização das informações que repercutem, pois, quando isso não ocorre, o impacto pode ser mais negativo do que positivo, principalmente quando constroem ou reforçam concepções equivocadas. No caso da inatividade física, isto é potencialmente grave no sentido de que pode legitimar a falta de investimentos em ações voltadas ao seu enfrentamento, pois, uma vez que a inatividade física passa a ser interpretada como uma mera questão de "preguiça", perde-se boa parte do sentido de se investir na modificação de diversos determinantes desse problema de saúde pública.

Atividade Motora; Estilo de Vida; Meios de Comunicação de Massa; Jornalismo Científico; Publicações Periódicas
Mathias Roberto Loch 1

Paulo Henrique Guerra 2,3

doi: 10.1590/0102-311X00223017

\author{
Correspondência \\ P. H. Guerra \\ Universidade Federal da Fronteira Sul. \\ Rodovia SC 484, km 02, Chapecó, SC 89815-899, Brasil. \\ paulo.guerra@uffs.edu.br \\ 1 Programa de Pós-graduação em Saúde Coletiva, Universidade \\ Estadual de Londrina, Londrina, Brasil. \\ 2 Universidade Federal da Fronteira Sul, Chapecó, Brasil. \\ 3 Grupo de Estudos e Pesquisas Epidemiológicas em Atividade \\ Física e Saúde, Universidade de São Paulo, São Paulo, Brasil.
}




\section{Introdução}

"A preguiça é a mãe do progresso. Se o homem não tivesse preguiça de caminhar, não teria inventado a roda" (Mário Quintana).

No cenário mundial, a inatividade física tem sido indicada como uma importante questão de saúde pública, principalmente em função da sua associação com uma série de doenças e mortalidade precoce ${ }^{1}$. No contexto brasileiro, muito pelo reconhecimento da sua alta prevalência no país $2,3,4$, nos últimos anos, importantes políticas públicas foram elaboradas e incluíram o enfrentamento a essa questão, como, por exemplo: a Política Nacional de Promoção da Saúde 5, Plano de Ações Estratégicas para o Enfrentamento das Doenças Crônicas Não Transmissíveis no Brasil 6 e o Programa Academia da Saúde 7, além de a atividade física ter sido reconhecida como um fator determinante e condicionante de saúde 8 .

À parte das diferenças em relação à forma de se definir a inatividade física, além de outras diferenças metodológicas existentes entre os estudos, evidências importantes apontam que, no Brasil, a inatividade física no tempo livre é determinada por uma série de variáveis e parece ser mais elevada em mulheres 3 , idosos 9 , negros 9 , pessoas com menor escolaridade 10 , menor renda 10 , menor capital social 11 e em pessoas que vivem em bairros que não possuem espaços para a prática de atividade física $3,10,12$.

Mesmo que o conhecimento popular sobre a importância da atividade física para a saúde remonte à época dos pensadores clássicos (tendo inclusive frases importantes atribuídas a Hipócrates), as primeiras pesquisas epidemiológicas nessa temática são recentes, datadas dos anos iniciais da década de 1950 13,14. Em vista do impacto dessas evidências, assim como sua contribuição para a promoção da saúde, a temática vem recebendo, além da atenção dos principais periódicos científicos internacionais de diversas áreas, a atenção de alguns dos principais veículos de comunicação em massa do Brasil e do mundo.

Uma rápida busca na Internet aponta que dados e indicadores de atividade física/inatividade física são comumente apresentados pela mídia do país, o que pode ser de grande importância, pois os veículos de comunicação em massa influenciam a vida de muitas pessoas e, inclusive, desempenham um papel central na projeção e legitimação do conhecimento 15 .

Entretanto, muitas vezes, o jornalismo direcionado à temática da saúde reforça padrões estéticos e comportamentais, por vezes, generalizantes e abordados de uma maneira descontextualizada 15,16. Luz et al. 17 observam que é muito comum que as capas de diversas revistas contenham imagens e palavras imperativas, geralmente expressas em tom de comando e que buscam orientar práticas de vida e de saúde, havendo um forte apelo pela "vida saudável”, especialmente a partir da alimentação e prática de atividade física. Os mesmos autores destacam que o tom imperativo tende a legitimar os comportamentos propostos. De uma maneira mais ampla, Moraes 18 considera que as notícias que abordam a saúde nos jornais são normalmente apresentadas de maneira pulverizada, descontínua e descontextualizada, prevalecendo o tratamento tópico e isolado. Além do mais, o mesmo autor também destaca que a direção ou intencionalidade da notícia nunca é explicitada.

Dessa forma, ao se reconhecer que, para uma considerável parcela da população, os meios de comunicação de massa ainda se configuram como a principal, quando não única, forma de acesso ao conhecimento científico, o presente ensaio teve o objetivo de apresentar elementos para a reflexão sobre discrepâncias entre as evidências científicas produzidas a respeito dos fatores associados e determinantes da inatividade física e as informações que são passadas pela mídia a respeito desse assunto, mais especificamente em um caso em que a "preguiça" foi utilizada como fator "explicativo" para os elevados níveis de inatividade física na população brasileira.

Para tal, utilizamos, como exemplos, um artigo publicado na revista científica Nature e reportagens veiculadas em dois canais de comunicação brasileiros de grande alcance, que o repercutiram. 


\section{Discrepâncias entre os discursos: 0 artigo de Althoff et al. e duas reportagens brasileiras}

Em 12 de julho de 2017, diversos canais de comunicação internacionais publicaram reportagens baseadas no artigo científico de Althoff et al. 19, intitulado Large-Scale Physical Activity Data Reveal Worldwide Activity Inequality, publicado na Nature. Em paralelo à repercussão internacional, realizamos uma leitura inicial do referido artigo, que nos deu as primeiras indicações de que, pelo menos, os títulos das matérias jornalísticas que estavam sendo publicadas tinham pouca ou nenhuma relação com suas informações e/ou conclusões.

Ao passo que essa repercussão chegou ao Brasil, sendo, inclusive, veiculada por alguns dos principais canais de comunicação do país, percebemos que tais incoerências foram também mantidas nos seus títulos. Entre os quais, o jornal $O$ Globo que publicou, em seu sítio eletrônico, às $12 \mathrm{~h} 14 \mathrm{~min}$ (horário de Brasília) do dia 12 de julho de 2017, a reportagem: Brasileiro Está Entre os Mais 'Preguiçosos' do Mundo 20, e a revista Veja que, no mesmo dia, às $17 \mathrm{~h} 19 \mathrm{~min}$, publicou, também em seu sítio eletrônico, a matéria Brasileiro É Um dos Mais Preguiçosos do Mundo 21.

Vale destacar que essas reportagens não foram as únicas publicadas no Brasil que repercutiram o artigo de Althoff et al. 19 e que a escolha dessas duas reportagens se deu de maneira intencional, em função da importância histórica desses dois veículos (influenciando, inclusive, na representação do que é ser brasileiro), do seu elevado número de leitores e em função da abordagem escolhida por elas nos seus títulos.

Desse modo, entendemos que essas reportagens seriam adequadas como "exemplos práticos" que seriam úteis no desenvolvimento das ideias apresentadas neste ensaio, não sendo intenção deste texto "monitorar" os demais veículos de comunicação brasileiros. Dessa forma, não podemos afirmar que todos os veículos de imprensa brasileira apresentaram abordagens semelhantes em relação à repercussão do artigo de Althoff et al. 19.

Por outro lado, destacamos que os principais meios de comunicação brasileiros pertencem a um pequeno número de famílias e que, geralmente, pouco diferem em suas abordagens, havendo pouco espaço para a apresentação de visões divergentes em diversos assuntos, inclusive políticos 22 , além de, muitas vezes, adotarem coberturas que não são condizentes com o interesse público 23.

A seguir, destacamos algumas das principais informações do estudo de Althoff et al. 19.

(i) Seu objetivo foi verificar a atividade física em nível mundial, assim como possíveis desigualdades associadas à classificação de renda.

(ii) A atividade física foi medida por meio de um aplicativo de acelerometria disponível para smartphones. Ao todo, o banco de dados contou com informações de 717.527 pessoas.

(iii) Para verificação das possíveis desigualdades, foram considerados os países com amostras de mais de mil participantes, a partir da classificação de renda do Banco Mundial. Dessa forma, fizeram parte do estudo pessoas de 32 países classificados como de alta renda ( $90 \%$ da amostra), dez países de renda média-alta e quatro países de renda baixa-média.

(iv) Países onde havia menor diferença na prática de atividade física entre os gêneros eram os lugares que, em geral, tinham menor prevalência de obesidade.

(v) Lugares com maiores níveis de "caminhabilidade" (do inglês walkability) apresentam menor diferença na prática de atividade física de homens e mulheres.

Consideramos como importantes implicações do texto: (1) a importância de se diminuir as iniquidades de gênero na prática de atividade física e (2) a importância do ambiente construído como "estratégia" para essa diminuição.

Entretanto, mesmo que o artigo traga relevantes evidências e implicações para a saúde pública, as reportagens analisadas passaram, pelo menos em seus títulos, muito distantes das conclusões ou recomendações originais, optando por focar na questão da "preguiça" como explicação central para o fato de o Brasil estar mal posicionado em um suposto ranking de prática de atividade física.

Em $O$ Globo 20, a palavra preguiça aparece entre aspas no título, ou seja, dando abertura para uma interpretação menos literal da expressão, mas sequer é mencionada no texto da reportagem. Assim, o título não representa o quê, de fato, é abordado no texto, que chega mesmo a ter um subtítulo que sugere um enfoque mais ampliado sobre a questão da inatividade física ("urbanismo como política de saúde pública"). No entanto, apesar de ser difícil estimar uma proporção, é muito provável que a maior 
parte das pessoas apenas leu o título da matéria, no qual a questão da "preguiça" como "explicação" para a elevada inatividade física na população brasileira fica explícita.

Veja ${ }^{21}$, por sua vez, não usou aspas no título da reportagem, mas sim no subtítulo: No Ranking que Avaliou os Países 'Mais Preguiçosos' o Brasil Ficou na 7a Posição, entre 46 Avaliados. Assim como no relato de $O$ Globo 20, a leitura feita pela revista a respeito do artigo de Althoff et al. 19 como mero estudo que fez um ranking dos "mais preguiçosos", é bastante "livre", inclusive, porque, no artigo original, em nenhum momento, a "preguiça” é mencionada como possível explicação para a diferença na prática de atividade física entre os países avaliados. Assim, fica evidente a incoerência entre o que diz o artigo científico publicado na Nature e o que dizem os títulos das duas reportagens analisadas.

Para além das análises entre os textos, vale mencionar que o dicionário on-line de português 24 define o substantivo "preguiça" como: "aversão ao trabalho; pouca disposição para trabalhar. lentidão em fazer qualquer coisa; moleza; morosidade”. Mesmo que as reportagens busquem contextualizar a preguiça pela ausência de atividade física, ao considerarmos as definições dos dicionários, parece claro que a expressão tem uma conotação histórica e bastante negativa no imaginário dos brasileiros.

Chaui 25 (p. 9-10) na Introdução de O Direito à Preguiça, de Paul Lafargue, menciona: "A preguiça, todos sabem, é um dos sete pecados capitais. (...) O laço que ata preguiça e pecado é um nó invisível que prende imagens sociais de escárnio, condenação e medo. É assim que aparecem para os brasileiros brancos as figuras do índio preguiçoso e do negro indolente, construídas no final do século XIX, quando o capitalismo exigiu a abolição da escravatura e substituiu a mão-de-obra escrava pelo imigrante europeu, chamado trabalhador livre (curiosa expressão numa sociedade cristã que não desconhece a Bíblia nem ignora que o trabalho foi imposto aos humanos como servidão!). É ainda no início deste século, do Jeca Tatu, o caipira ocioso devorado pelos vermes enquanto a plantação é devorada pelas saúvas. Nesse imaginário, 'a preguiça é a mãe de todos os vícios'e nele vêm inscreverse, hoje, o nordestino preguiçoso, a criança de rua vadia (...), o mendigo (...). É ela, enfim, que força o trabalhador desempregado a sentir-se humilhado, culpado e um pária social".

Ainda sobre a acepção do termo preguiça no Brasil, é importante citarmos o personagem Macunaíma, de Mário de Andrade, que, mesmo tendo nascido índio, pode, de certa forma, representar um olhar crítico à complexidade étnica, cultural, social e moral da formação do povo brasileiro. Tendo como sua marca registrada a frase “Ai que preguiça!", seus esforços direcionam-se apenas para um único fim: seu benefício, independente se à custa do bem-estar de outrem. Na adaptação cinematográfica da obra, Macunaíma, interpretado por Grande Otelo, nasceu feio e desajustado, elementos os quais, por outro lado, podem sugerir, ou mesmo fomentar, um perfil do típico "preguiçoso brasileiro".

Tomando esses exemplos e outros que estão incutidos no imaginário do brasileiro e poderiam ser citados, fica clara a conotação negativa da preguiça no contexto nacional. Mesmo se ponderando que não fora objetivo das matérias analisadas uma reflexão mais profunda dos motivos pelos quais o brasileiro é fisicamente inativo - erroneamente caracterizado como preguiçoso nos dois títulos -, entendemos que não podem ser desconsiderados a importância e os valores atribuídos à preguiça nas esferas histórica, cultural e social do país.

Ademais, como destacam Castiel \& Álvarez-Dardet Diaz 26 (p. 25) "os discursos de saúde nunca se referem tão somente a dimensões da saúde. Se tais discursos significam modos de pensar, escrever, falar sobre saúde e suas práticas, é preciso situá-los em determinados momentos históricos e saber as razões por que se legitimam ao acompanharem a ordem econômica, política e social onde são gerados, sustentados e replicados a se ajustarem a ela”. É fundamental se destacar que essa observação serve não apenas para o discurso jornalístico, mas também para o discurso científico.

No caso da atividade física e, de uma maneira mais ampla, no "discurso do estilo de vida", alguns autores apontam que não é incomum encontrar, no discurso acadêmico/científico, olhares simplistas, moralistas e descontextualizados 26,27,28,29, que, muitas vezes, ignoram as condições de vida das pessoas e que limitam a saúde à questão dos comportamentos relacionados à saúde e com aproximações superficiais de movimentos mais amplos, como o Movimento da Promoção da Saúde e todo o acúmulo a respeito dos determinantes sociais do processo saúde-doença 30,31.

De qualquer modo, chamou-nos a atenção o fato de que, nas reportagens utilizadas neste texto, não são citados especialistas brasileiros, o que talvez pudesse gerar um melhor entendimento sobre nossas especificidades. No entanto, considerando-se que a linguagem acadêmica/científica em alguns casos não é "isenta” de interpretações superficiais e descontextualizadas, vale ressaltar que essa consulta, não garantiria, por si só, esse melhor entendimento. 
Cabe mencionar um fato importante que não foi observado na discussão do ranking apresentado pelas reportagens analisadas. Dos vinte países mais bem ranqueados no número de passos (os "menos preguiçosos”, segundo as chamadas das reportagens), 17 pertencem ao grupo dos países cuja economia está classificada como de alta renda pelo Banco Mundial (85\%), enquanto, dos dez países piores "ranqueados" (estando o Brasil nesse rol), apenas dois são classificados como economias de alta renda (Arábia Saudita e Catar).

Consideramos que duas interpretações totalmente opostas são possíveis. Na primeira, a preguiça está associada à pobreza. Nessa interpretação, o ranking seria coerente com a ideia de que os países mais "preguiçosos" são aqueles em que as pessoas trabalham/produzem menos e que, por isso, são mais pobres. A outra leitura, que é a que nos parece mais adequada, é que a prática de atividade física, especialmente no tempo livre, está bastante associada a indicadores socioeconômicos pessoais e do próprio contexto, o que, dessa forma, permitindo-nos refleti-la mais como a "consequência" de um contexto desfavorável do que "causa".

Uma ponderação importante precisa ser realizada: a interpretação da "preguiça” como causa para a inatividade física não foi somente feita nos veículos de comunicação brasileiros. Encontramos abordagens semelhantes em veículos dos Estados Unidos, Austrália, Reino Unido, Hong Kong e Espanha. No caso da British Broadcasting Corporation (BBC), por exemplo, houve, inclusive, uma readequação do título da reportagem inicial, que passou de How Lazy is Your Country para Do You Live in the World's Least Active Country?

Acreditamos que dificilmente os jornalistas de $O$ Globo e Veja tenham lido o artigo de Althoff et al. 19; sendo mais provável que tenham se baseado exclusivamente em alguma reportagem internacional para repercutirem o assunto no Brasil, mantendo-se a inadequação por eles disseminada. De qualquer modo, essa é apenas uma hipótese nossa, observando-se a proximidade entre os títulos, o que, por outro lado, também não isenta os profissionais que realizaram as reportagens de suas responsabilidades, bem como os veículos que as publicaram.

\section{Comentários finais}

O Brasil é um importante polo científico sobre a atividade física e um dos pioneiros em políticas públicas voltadas a sua promoção 5,6,7,32. O artigo de Althoff et al. ${ }^{19}$ gerou grande repercussão mundial na mídia e apresenta importantes conclusões que podem ser importantes no direcionamento das ações de promoção da atividade física, inclusive, fortalecendo a ideia de que sua prática é influenciada por variáveis mais amplas, até mesmo ambientais 33,34 .

No entanto, duas reportagens brasileiras 20,21, que repercutiram o estudo de Althoff et al. ${ }^{19}$, publicadas em veículos de comunicação que têm um elevado número de leitores, adotaram, especialmente em seus títulos ou subtítulos, uma abordagem que consideramos inadequada, simplificadora e incoerente tanto para com o próprio estudo quanto para com os determinantes da inatividade física no país. Acreditamos que os meios de comunicação em massa, em suas distintas formas de manifestação, têm um papel fundamental na difusão de informações para a sociedade.

No entanto, quando essa difusão não é acompanhada de uma devida contextualização, ela pode ser mais negativa do que positiva, principalmente quando constrói ou reforça concepções que são equivocadas, como no caso da ideia da "preguiça" explicar a inatividade física da população.

Nessa questão específica, isso seria especialmente grave, no sentido de que pode, inclusive, legitimar a falta de investimentos em ações voltadas à promoção da atividade física, pois, nessa acepção, uma vez que a inatividade física é uma mera questão de "preguiça", perde-se boa parte do sentido de se investir em importantes aspectos, como melhoria dos ambientes construídos, diminuição da violência e dos diversos tipos de iniquidades, investimento em programas de atividade física em geral, melhora da formação dos profissionais de saúde envolvidos nesse tema etc. Enfim, na modificação de diversos determinantes da prática de atividade física.

Dado esse contexto, é fundamental que a comunidade científica fique atenta às repercussões de suas produções quando essas são divulgadas pelos diversos meios de comunicação, bem como busque ser cuidadosa e criteriosa na escolha das expressões a serem utilizadas e na indicação da aplicação das suas evidências, que devem sempre considerar a realidade objetiva das pessoas e populações. Conside- 
ramos essa recomendação importante porque reconhecemos que não é incomum que a simplificação e a inadequação partam, algumas vezes, da própria comunidade científica.

Vale mencionar que a preocupação com a abordagem dada pelos meios de comunicação ao "tema" saúde não é nova 15,16,17,18,23,35,36,37,38,39,40,41,42, havendo importantes esforços direcionados a uma sistematização do monitoramento das publicações realizadas pelos veículos de comunicação, como é o caso do Observatório Saúde na Mídia, da Fundação Oswaldo Cruz, que busca, entre outros objetivos, realizar o acompanhamento diário das informações sobre saúde publicadas em jornais nacionais e investigar os sentidos da saúde apresentados pela imprensa.

No caso específico da inatividade física, indicamos que é urgente o aprofundamento da sua natureza complexa, multifatorial e intersetorial, de modo que noções simplistas e descontextualizadas, que não são sustentadas pelas diversas evidências científicas já acumuladas a respeito dos fatores que explicam seus elevados níveis, sejam superadas e deixem de ser divulgadas para a população em geral.

\section{Colaboradores}

M. R. Loch participou da concepção da ideia central da pesquisa, levantamento e análise dos dados, desenvolvimento e revisão do texto. P. H. Guerra colaborou no levantamento e análise dos dados, desenvolvimento e revisão do texto.

\section{Referências}

1. Lee IM, Shiroma EJ, Lobelo F, Puska P, Blair SN, Katzmarzyk PT, et al. Impact of physical inactivity on the world's major non-communicable diseases. Lancet 2012; 380:219-29.

2. Departamento de Vigilância de Doenças e Agravos não Transmissíveis e Promoção da Saúde, Secretaria de Vigilância em Saúde, Ministério da Saúde. Vigitel Brasil 2016: vigilância de fatores de risco e proteção para doenças crônicas por inquérito telefônico: estimativas sobre frequência e distribuição sociodemográfica de fatores de risco e proteção para doenças crônicas nas capitais dos 26 estados brasileiros e no Distrito Federal em 2016. Brasília: Ministério da Saúde; 2017.

3. Florindo AA, Hallal PC, Moura EC, Malta DC. Prática de atividades físicas e fatores associados em adultos, Brasil, 2006. Rev Saúde Pública 2009; 43 Suppl 2:65-73.

4. Mielke GI, Malta DC, Sá GBAR, Reis RS, Hallal PC. Diferenças regionais e fatores associados à prática de atividade física no lazer no Brasil: resultados da Pesquisa Nacional de Saúde-2013. Rev Bras Epidemiol 2015; 18 Suppl 2:158-69.

5. Ministério da Saúde. Portaria no 2.446, de 11 de novembro de 2014. Redefine a Política Nacional de Promoção da Saúde (PNPS). Diário Oficial da União 2014; 12 nov.
6. Departamento de Análise de Situação de Saúde, Secretaria de Vigilância em Saúde, Ministério da Saúde. Plano de ações estratégicas para o enfrentamento das doenças crônicas não transmissíveis (DCNT) no Brasil 2011-2022. Brasília: Ministério da Saúde; 2011.

7. Ministério da Saúde. Portaria no 2.681, de 7 de novembro de 2013. Redefine o Programa Academia da Saúde no âmbito do Sistema Único de Saúde (SUS). Diário Oficial da União 2013; 8 nov.

8. Ministério da Saúde. Lei no 12.864 , de 24 de setembro de 2013. Altera o caput do art. 3o da Lei no 8.080, de 19 de setembro de 1990, incluindo a atividade física como fator determinante e condicionante da saúde. Diário Oficial da União 2013; 25 set.

9. Programa das Nações Unidas para o Desenvolvimento. Relatório de desenvolvimento humano nacional - movimento é vida: atividades físicas e esportivas para todas as pessoas. Brasília: Programa das Nações Unidas para o Desenvolvimento; 2017.

10. Pitanga FJG, Matos SMA, Almeida MC, Molina MCB, Aquino EL. Factors associated with leisure time physical activity among ELSABrasil participants: ecological model. Prev Med 2016; 90:17-25. 
11. Loch MR, Souza RKT, Mesas AE, MartinezGómez D, Rodríguez-Artalejo F. Relationship between social capital indicators and lifestyle in Brazilian adults. Cad Saúde Pública 2015; 31:1636-47.

12. Florindo AA, Barrozo LV, Cabral-Miranda W, Rodrigues EQ, Turrell G, Goldbaum M, et al. Public open spaces and leisure-time walking in Brazilian adults. Int J Environ Res Public Health 2017; 14:E553.

13. Morris JN, Heady JA. Mortality in relation to the physical activity of work: a preliminary note on experience in middle age. $\mathrm{Br} \mathrm{J}$ Ind Med 1953; 10:245-54.

14. Morris JN, Heady JA, Raffle PAB, Roberts CG, Parks, JW. Coronary heart-disease and physical activity of work. Lancet 1953; 265:1053-7.

15. Serra GMA, Santos EM. Saúde e mídia na construção da obesidade e do corpo perfeito. Ciênc Saúde Coletiva 2003; 8:691-701.

16. Oliveira VC. Os sentidos da saúde nas mídias jornalísticas impressas. RECIIS (Online) 2013; 6(4). https://www.reciis.icict.fiocruz.br/index. $\mathrm{php} / \mathrm{reciis} /$ article/view/622.

17. Luz MT, Ferla AA, Machado AS, Alba RD. Retórica na divulgação científica do imaginário de vida e saúde: uma proposta metodológica de análise. Interface (Botucatu) 2017; 21:33347.

18. Moraes NA. Comunicação, sentidos e saúde. In: Conselho Nacional de Saúde; Ministério da Saúde, organizadores. Coletânea de comunicação e informação em saúde para o exercício do controle social. Brasília: Ministério da Saúde; 2007. p. 61-6.

19. Althoff T, Sosic R, Hicks JL, King AC, Delp SL, Leskovec J. Large-scale physical activity data reveal worldwide activity inequality. Nature 2017; 547:336-9.

20. Matuura S. Brasileiro está entre os mais 'preguiçosos' do mundo. O Globo 2017; 12 jul. https://oglobo.globo.com/sociedade/saude/ brasileiro-eta-entre-os-mais-preguicosos-domundo-21579902.

21. Brasileiro é um dos mais preguiçosos do mundo. Veja 2017; 12 jul. https://veja.abril.com.br/ saude/brasileiro-e-um-dos-mais-preguicososdo-mundo/.

22. Reporters Without Borders. Brazil: the country of thirty Berlusconis. https://rsf.org/en/re ports/brazil-country-thirty-berlusconis (acessado em 18/Dez/2017).

23. Rocha RL. Os negócios da mídia e a comunicação da saúde. Cad Saúde Pública 2016; 32:e00000616.

24. Dicionário on-line de Português. https://www. dicio.com.br/ (acessado em 20/Dez/2017).

25. Chaui M. Introdução. In: Lafargue P, organizador. $\mathrm{O}$ direito à preguiça. São Paulo: Editora Hucitec/Universidade Estadual Paulista; 1999. p. 9-56.

26. Castiel LD, Álvarez-Dardet Diaz C. A saúde persecutória: os limites da responsabilidade. Rio de Janeiro: Editora Fiocruz; 2007.
27. Castiel LD, Ferreira MS, Moraes DR. Os riscos e a promoção do autocontrole na saúde alimentar: moralismo, biopolítica e crítica parresiasta. Ciênc Saúde Coletiva 2014; 19:1523-32.

28. Ferreira MS, Castiel LD, Cardoso MHCA. A patologização do sedentarismo. Saúde Soc 2012; 21:836-47.

29. Fraga AB. El sedentarismo es... Saúde Soc 2016; 25:716-20.

30. Knuth AG, Loch, MR. "Saúde é o que interessa, o resto não tem pressa"? Um ensaio sobre educação física e saúde na escola. Rev Bras Ativ Fís Saúde 2014; 19:429-40.

31. Souza RKT, Loch MR. Promoção da saúde. In: Andrade SM, Cordoni Jr. L, Carvalho BG, González AD, Silva AMR, organizadores. Bases da saúde coletiva. 2a Ed. Londrina: Eduel; 2017. p. 29-46.

32. Hallal PC. Atividade física e saúde no Brasil: pesquisa, vigilância e políticas. Cad Saúde Pública 2014; 30:2487-9.

33. Bauman AE, Reis RS, Sallis JF, Wells JC, Loos RJ. Martin BW. Correlates of physical activity: why are some people physically active and others not? Lancet 2012; 380:258-71.

34. Sallis JF, Cerin E, Conway TL, Adams MA, Frank LD, Pratt M, et al. Physical activity in relation to urban environments in 14 cities worldwide: a cross-sectional study. Lancet 2016; 387:2207-17.

35. Lopes B, Nascimento J. Saúde e imprensa: o público que se dane. Rio de Janeiro: Editora Mauad; 1996.

36. Tabakman R. A saúde na mídia: medicina para jornalistas, jornalismo para médicos. São Paulo: Lummus Editorial; 2013.

37. Lerner K, Sacramento I. Saúde e jornalismo: interfaces contemporâneas. Rio de Janeiro: Editora Fiocruz; 2014

38. D’Avilla C, Trigueiros U. Comunicação, mídia e saúde: novos agentes, novas agendas. Rio de Janeiro: Luminatti Editora; 2017.

39. Moraes R, Oliveira-Costa MS, Mendonça AVM. De que saúde pública estamos falando? Um olhar sobre os discursos jornalísticos no Correio Braziliense em 2016. Revista Latinoamericana de Ciências de la Comunicación 2017; 14:326-41.

40. Cavaca A, Emerich T, Lerner K. Observatórios de Saúde na Mídia: dispositivos de análise crítica em Comunicação e Saúde. Rev Bras Pesqui Saúde 2016; 18:4-5.

41. Cavaca AG, Emmerich TB, Santos Neto ET, Vasconcellos-Silva PR, Emmerich A. Diseases neglected by the media in Espírito Santo, Brazil in 2011-2012. PLoS Negl Trop Dis 2016; 10:e0004662.

42. Lerner K, Gradella P. Mídia e pandemia: os sentidos do medo na cobertura de influenza H1N1 nos jornais cariocas. Revista ECO-Pós 2011; 14:33-54. 


\section{Abstract}

This essay aims to suggest food for thought on discrepancies between scientific evidence on physical inactivity and the associated factors and determinants and information communicated by the media on this topic. We specifically examine a case in which "laziness" was used as the "explanatory" factor for the high levels of physical inactivity in the Brazilian population. We use as examples a scientific article published in Nature and two news stories published in mainstream Brazilian media channels (Veja magazine and O Globo newspaper) that commented on the former. There were important inconsistencies between the scientific content in the Nature article and the news stories, which took a simplistic approach to a real and complex problem (high levels of physical inactivity in Brazil), suggesting that "laziness" explains why Brazil ranks so poorly in the world in terms of physical activity. We highlight the need for mass communications media to better contextualize the information they republish, because otherwise the impact can be more negative than positive, especially when they build or reinforce misconceptions. This is particularly serious in the case of physical inactivity, since such news coverage can lend legitimacy to the lack of investments in measures to deal adequately with the problem. If physical inactivity is interpreted merely as a matter of "laziness", it makes much less sense to invest in modifying various determinants of this serious public health problem.

Motor Activity; Life Style; Mass Media; Scientific Journalism; Periodicals

\section{Resumen}

Este ensayo pretende presentar elementos para la reflexión sobre las discrepancias entre las evidencias científicas, producidas respecto a factores asociados y determinantes de la inactividad física, y la información que trasmiten los medios de comunicación respecto a este asunto, pero especificamente en un caso en el que la "pereza" se utilizó como factor "explicativo" para los elevados niveles de inactividad física en la población brasileña. Utilizamos, como ejemplos, un artículo científico publicado en Nature y dos reportajes difundidos a través de canales de comunicación brasileños de gran alcance (revista Veja y periódico O Globo), que lo difundieron. Se observaron importantes incoherencias entre el contenido del artículo de $\mathrm{Na}$ ture, y los títulos de los reportajes analizados, de manera que estos simplificaron un problema real y complejo (los altos niveles de inactividad física en Brasil), dando a entender que la "pereza" sería el motivo por el que Brasil estaría mal posicionado en un ranking internacional de práctica de actividad física. Destacamos la necesidad de que los medios de comunicación de masas busquen una mejor contextualización de la información que difunden puesto que, cuando esto no sucede, el impacto puede ser más negativo que positivo, principalmente cuando construyen o refuerzan concepciones equivocadas. En el caso de la inactividad física, esto es potencialmente grave. En el sentido de que puede legitimar la falta de inversión en acciones dirigidas a su combate, ya que, una vez que la inactividad física pasa a ser interpretada como una mera cuestión de "pereza", se pierde buena parte del sentido en invertir para la modificación de diversos determinantes de este problema de salud pública.

Actividad Motora; Estilo de Vida; Medios de Comunicación de Masas; Periodismo Científico; Publicaciones Periódicas
Recebido em 26/Dez/2017

Versão final reapresnetada em 29/Jun/2018

Aprovado em 17/Ago/2018 